\title{
Rhabdomyolysis associated to glossopharyngeal edema: A rare side effect of Diclofenac
}

\author{
Hicham Bakkali $^{1}$, *, Lahcen Belyamani ${ }^{1}$, Salahedine Massou ${ }^{2}$, Loukmane Elwartiti $^{2}$, \\ Khalil Aboulaala $^{2}$, Hicham Balkhi ${ }^{2}$, Charqui Haimeur ${ }^{2}$ \\ ${ }^{1}$ Department of Emergency, Military Training Hospital Med V, Rabat, Morocco \\ ${ }^{2}$ Department of Anesthesiology and Critical Care, Military Training Hospital Med V, Rabat, Morocco \\ ${ }^{3}$ Faculty of Medicine and Pharmacy, University Med V, Rabat, Morocco
}

Email address:

hbakkali@ymail.com (H. Bakkali)

\section{To cite this article:}

Hicham Bakkali, Lahcen Belyamani, Salahedine Massou, Loukmane Elwartiti, Khalil Aboulaala, Hicham Balkhi, Charqui Haimeur. Rhabdomyolysis Associated to Glossopharyngeal Edema: A Rare Side Effect of Diclofenac. American Journal of Clinical and Experimental Medicine. Vol. 2, No. 6, 2014, pp. 161-164. doi: 10.11648/j.ajcem.20140206.18

\begin{abstract}
Rhabdomyolysis is a widely used non steroid anti-inflammatory drug. Rhabdomyolysis after taking Diclofenac is rarely reported, and the association to a glossopharyngeal edema has never been discussed. Here is a case of rhabdomyolysis associated to glossopharyngeal edema after taking $200 \mathrm{mg}$ of Diclofenac. The patient was first diagnosed with para phenyl di-amine intoxication but the toxicological examinations were negative. The patient outcome was good after symptomatic treatment.
\end{abstract}

Keywords: Diclofenac, Intoxication, Rhabdomyolysis, Glossopharyngeal Edema

\section{Introduction}

Acute rhabdomyolysis is a clinical and laboratory syndrome resulting from the breakdown of skeletal muscle, with the release of intracellular contents into the circulatory system, which can cause potentially lethal complications. Rhabdomyolysis can be a consequence of various causes, which include genetic andmetabolic myopathies, trauma, infections, electrical injuries, hyperthermia, autoimmune disorders; Drugs are one of the common causes. Rhabdomyolysis secondary to Diclofenac was described as sporadic cases in the literature. However, the association of a glossopharyngeal edema to severe rhabdomyolysis after administring Diclofenac has never been discussed before. In this paper we will discuss the peculiarities of the clinical picture, the difficulty of etiologic diagnosis and the differential diagnosis problem that arises mainly with PPDA (Para-phenylene di-amine) intoxication, frequent in Morocco.

\section{Observation}

A 50 year-old patient, addicted smoker and allergic to aspirin, was admitted to the emergency department with an acute respiratory distress syndrome. The symptoms appeared seven hours before the admission, manifested by a cervico-facial edema a few hours after taking four 50mg tablets $(200 \mathrm{mg})$ of Diclofenac Potassium. According to the clinical examination, the patient was fully alert and oriented he was dyspneic, respiratory rate 25 breaths per minute, and an oxygen saturation of $93 \%$. He had a cervico-facial and glossopharyngeal edema causing a tongue protrusion. His Glasgow Coma Scale (GCS) score was 15. His body temperature was $37,5 \mathrm{C}$, blood pressure was $135 / 85 \mathrm{mmHg}$, pulse was 85 beats per minute He didn't present any skin lesion. On chest auscultation the patient had normal vesicular breathing sounds. Cardiac auscultation was normal. Abdominal examination was normal too, no hepatomegaly or splenomegaly was appreciated.

Nasotracheal fiberoptic intubation was thus urgent. The patient was then accepted in intensive care with monitoring and conditioning. Urinary catheter showed some dark urine. Initial laboratory tests found the following: 7.30 of $\mathrm{PH} ; 36$ $\mathrm{mmHg}$ of $\mathrm{PCO} 2 ; 232 \mathrm{mmHg}$ of $\mathrm{PaO} 2 ; 21 \mathrm{mmol} / 1$ of $\mathrm{HCO}^{-}$; $2,2 \mathrm{mmol} / \mathrm{l}$ of lactates; $3,5 \mathrm{mmoles} / 1$ of potassium; $0,33 \mathrm{~g} / \mathrm{l}$ of urea, and $21 \mathrm{mg} / \mathrm{l}$ of creatinine. The rhabdomyolysis test showed $812 \mathrm{IU} / 1$ of ASAT, $1184 \mathrm{IU} / 1$ of $\mathrm{LDH}$, and $0,321 \mu \mathrm{g} / \mathrm{l}$ of Troponins (six times the normal rate). The Creatine Kinase 
(CK) were out of range. Chest X-ray and ECG tests showed no particular results. The patient was first diagnosed with PPDA intoxication. The toxicological examinations of the gastric fluid, blood and urine were negative. The patient was treated with volume expander, alkalizing and forced diuresis. The electrolytes and arterial blood gases were monitored twice a day. The latter showed high occurrence of mixed acidosis with hyperkalemia. An anuric acute renal failure developed, requiring an extra-renal purification. A few days later, he was symptomatically improved, with electrolytes within normal limits, and liver function tests and CK levels trending down. An extubation was possible after the edema regression in the fourth day of hospitalization. The extra-renal replacement therapy continued until the amount of creatinine reached a normal level by the $25^{\text {th }}$ day.

\section{Discussion}

Diclofenac is a widely used nonsteroidal anti-inflammatory drug (NSAID); Diclofenac Potassium is absorbed more rapidly with an earlier peak compared to Diclofenac Sodium. It prevents the synthesis of prostaglandins by inhibiting cyclooxygenase responsible for major side effects. NSAIDs have other functions as well (uncoupling oxidative phosphorylation, inhibiting phosphodiesterases, blocking the activity of lysozyme and the migration of leukocytes to sites of inflammation, antagonizing prostaglandin receptors) as described in the literature $[1,2]$. The decoupling of oxidative phosphorylation can deplete the cell's ATP reserves, which is considered as the mechanism responsible for several cases of drug-induced rhabdomyolysis [3]. Some people with a family history of myopathies can particularly have this type of rhabdomyolysis [3]. The patient described here has no family history of myopathy.

Rhabdomyolysis is a pathological syndrome caused by skeletal muscle cell damage which affects the integrity of the cellular membrane and leads to the release of toxic intracellular constituents into the blood circulation. Its main causes include trauma, ischemia, drugs, toxins, metabolic disorders, and infections. In the past, the more common causes of acute rhabdomyolysis were from crush injuries during wartime and natural disasters.

More recently, as noted in one published series, drugs and alcohol have become frequent causative agents in up to $81 \%$ of cases of rhabdomyolysis [4].

Rhabdomyolysis after taking Diclofenac alone (or other NSAIDs) is rarely reported. Only four cases are reported in the literature $[5,6,7,8]$. Although rhabdomyolysis has been reported in many observations when therapy included Diclofenac, no particular drug causality was established. The particularity of the observation described in this paper is the rapid manifestation of the syndrome (the symptoms appeared in less than seven hours after administering Diclofenac) and the absence of any other possible factor (effort, seizures, trauma, electric shock, burn, metabolic disorder).

The therapeutic dose of Diclofenac is 100 to $150 \mathrm{mg}$ /day for adults. Some prescriptions tolerate a maximum dose of 200 mg. The toxic dose is not specified. Usually we assume a threshold of 10 times the therapeutic dose for adults and 5 times for children. The patient described here took $200 \mathrm{mg}$ in less than 24 hours. It's thus a side effect rather than intoxication. Similar dose, of $200 \mathrm{mg}$, is reported in one of the three other cases discussed in the literature [6]. Rhabdomyolysis is defined as a clinical and biochemical syndrome in which leakage of intracellular myocyte contents are released into the extracellular fluid and circulation. Myoglobin is a protein that functions as an important oxygen carrier that maintains the ability of red muscles to consume oxygen. The normal level of myoglobin in serum is 3 to 80 $\mu \mathrm{g} / \mathrm{L}$. The serum level of myoglobin is dependent upon the glomerular filtration rate. When $100 \mathrm{~g}$ of muscle tissue has been injured, the serum proteins reach the saturation level. All myoglobin above $230 \mathrm{mg} / \mathrm{L}$ is filtered through the glomerulus. The presence of myoglobin in the urine will produce a dark red-brown pigmentation if the level exceeds $1 \mathrm{~g} / \mathrm{L}$.

At or below a $\mathrm{pH}$ of 5.6, myoglobin dissociates into ferrihemate and globulin. Ferrihemate causes a direct deterioration of renal function, impairment of renal tubular transport mechanisms, and cell death. Myoglobinuric renal failure may be explained by a direct nephrotoxicity due to ferrihemate, tubular obstruction by precipitation of myoglobin casts, and alterations in glomerular filtration rate. Myoglobin can be detected in the urine in levels as low as 5 to $10 \mathrm{mg} / \mathrm{L}$ with a dipstick method that uses the orthotolidine reaction. Hemoglobinuria may also cause a positive orthotolidine reaction; however, the plasma will be pink, and red blood cells will be present on the microscopic evaluation. Myoglobinuria may precede and resolve prior to an increase in creatine kinase (CK) due to a short half-life of 1 to 3 hours. Therefore, a negative orthotolidine reaction does not rule out rhabdomyolysis [9].

Human tissues are composed of three different $\mathrm{CK}$ isoenzymes. The predominant isoenzyme is skeletal muscle and cardiac tissue is CK-MM. The function of CK is to convert myocyte creatine phosphate into high-energy phosphate groups (adenosine triphosphate) used in energy requiring reactions. The release of $\mathrm{CK}$ into the serum may reach levels up into the hundreds of thousands. Degradation of approximately $200 \mathrm{~g}$ of muscle can cause an increase in serum CK. Therefore, total serum $\mathrm{CK}$ is the most sensitive biochemical indicator of rhabdomyolysis. Serum concentration begins to increase 2 to 12 hours after the initial muscle injury and will peak at 3 to 5 days. Thus it is possible for myoglobinuria to be resolved prior to an elevated serum CK. Therefore, it is important to remember that in the initial acute rhabdomyolysis syndrome, serum CK may be normal.

When massive myocyte breakdown of cell membranes occurs, other intracellular constituents are released besides myoglobin and CK. A substantial amount of fluid can accumulate within the affected muscles causing elevated pressures in the fascial compartments. Intracellular potassium is released that can cause a significant hyperkalemia. Approximately $150 \mathrm{~g}$ of muscle necrosis will release more than $15 \mathrm{mmol}$ of potassium. The resulting hyperkalemia may 
increase the risk for cardiac arrhythmias and complicate an existing acute renal failure. In the beginning phases of rhabdomyolysis, calcium accumulates within the muscle with a resulting hypocalcemia. During the later stages, calcium is mobilized from the necrotic muscle tissue and results in hypercalcemia. Release of phosphate further contributes to the hypocalcemia by forming a calcium phosphate product that is deposited in the muscle tissue. Other metabolic abnormalities include metabolic acidosis, hyperuricemia, elevated lactate dehydrogenase, aldolase, creatinine, uric acid, urea, and amino transferases.

Even though the final diagnosis of rhabdomyolysis is established by laboratory findings, alertness to the syndrome is essential for prompt diagnosis. The clinical spectrum of rhabdomyolysis is rather wide. The typical triad of symptoms involves muscular pain, weakness, and reddish- brown urine. In $50 \%$ of the cases, the pain is focused on the central muscle groups (thighs, shoulders).

However, more than half of the patients do not report muscular symptoms. The reddish-brown color of urine is due to myoglobinuria and constitutes a powerful diagnostic element. Nonetheless, it is observed in approximately half of the cases and its absence does not exclude the syndrome. Muscular stiffness and seizures have also been reported. In more severe cases of rhabdomyolysis, general symptoms, such as malaise, fever, tachycardia, nausea, and vomiting, can be observed.

In the clinical examination, muscles may be swollen and sensitive during palpation, while changes in the color of skin compatible with compression necrosis may occur [9].

Complications can include rhabdomyolysis with acute renal failure, metabolic instability (acidosis, hyperkalemia, hyperphosphatemia, hyperuricemia, hypocalcemia), disseminated intravascular coagulation, compartment syndrome and peripheral neuropathy. The cervico-facial and glossopharyngeal edema (Quincke's edema) is an effect of the anaphylactic reaction and may be caused by a nonsteroidal anti-inflammatory drug. The clinical picture is so rich and has other clinical manifestation of the following types: rush cutaneous, urticaria, bronchospasm with sibilants and hypotension that can lead to a shock, which was not the case for the patient described here, suggesting thus another type of edema with a mechanism that may be similar to that observed in cases of PPDA intoxication. This edema was not described in the other cases of rhabdomyolysis associated with Diclofenac. Despite the lack of PPDA and muscle edema or a compartment syndrome very frequent in this intoxication, only the toxicological examination helped disregard this diagnosis.

Poisoning with PPDA presents with the characteristic features of severe angioneurotic edema, rhabdomyolysis and intravascular hemolysis with hemoglobinuria culminating in acute renal failure. Kallel et al. studied 19 patients with PPD intoxication in Tunisia over a six-year period.

Clinical symptoms were dominated by cervicofacial edema (79\%), chocolate-brown colored urine (74\%), upper airway tract edema $(68.4 \%)$, oliguria $(36.8 \%)$, muscular edema (26.3\%) and shock (26.3\%). Rhabdomyolysis and metabolic acidosis were seen in all the patients. ARF was seen in $47.4 \%$ and hyperkalemia in $26.3 \%$ [10].

PPDA can bring about rhabdomyolysis by promoting calcium release and leakage of calcium ions from the smooth endoplasmic reticulum, followed by continuous contraction and irreversible change in the muscle's structure.

Rhabdomyolysis is the main cause of acute renal failure and the morbidity and mortality are high once renal failure develops. Hypovolemia and the direct toxic effects of PPDA or its metabolites on the kidneys also contribute. Histologic changes of acute tubular necrosis have been described in PPDA poisoning.

The respiratory syndrome following the ingestion of PPDA is represented by asphyxia and respiratory failure secondary to inflammatory edema involving cricopharynx and larynx [11].

In any acute life-threatening ingestion or illness, the airway, ventilation, and perfusion should be the initial priority. Thereafter, the goal of treatment of rhabdomyolysis is to cease muscle destruction. The prevention of increased agitation, seizures, and abnormal movements must be attempted with pharmacologic agents. Treatment of hyperthermia is essential using external cooling measures and controlling for muscular hyperactivity with benzodiazepines. Electrolyte abnormalities that must be corrected are hyponatremia, hypernatremia, hyperglycemia, hypocalcemia, and decreased phosphorous. Alkalinization of urine and mannitol has shown to be effective in some patients with acute renal failure.

However, there is no standard protocol in the literature, and the bicarbonate may potentiate an already existing hypocalcemia. In the case of drug-induced rhabdomyolysis, eliminating the exposure of the toxic agent may be the only treatment[4].

In case of an anuric acute renal failure, an extra-renal replacement shall be required till we get a normal renal function. Our patient was treated with volume expander, alkalizing and forced diuresis, an anuric acute renal failure was developed, requiring an extra-renal purification which has been continued until the amount of creatinine reached a normal level by the $25^{\text {th }}$ day.

The prognosis of rhabdomyolysis depends on the complications resulting from the rhabdomyolysis and the underlying cause. When treated early and aggressively, an episode of rhabdomyolysis has an excellent prognosis. The mortality rate from rhabdomyolysis is about $8-10 \%$ [12]. Prognosis is substantially worse if ARF develops.

\section{Conclusion}

Rhabdomyolysis is a serious and potentially life threatening condition. Tere are a number of possible factors that may lead to acute rhabdomyolysis, and many patients present with multiple causes.

Rhabdomyolysis secondary to Diclofenac is a rare side effect that can appear due to sub-therapeutic doses. It is a serious and unpredictable adverse event that may happen. Its gravity requires awareness of any drug-induced rhabdomyolysis. It needs early detection and prompt intervention. 


\section{References}

[1] BOOTHE D.M. Controlling inflammation with non steroidal anti inflammatory drugs. Vet. Med., 1989, 84: 875-883.

[2] KORE A.M. Toxicology of non steroidal anti inflammatory drugs. Vet. Clin. of North Am. 1990, 20(2): 419-430.

[3] Hopkins P. M.. Anaesthesia and the sex-linked dystrophies: between a rock and a hard place. British Journal of Anaesthesia. 2010,104 (4): 397-400.

[4] 4 George D. Giannoglou , Yiannis S. Chatzizisis, Gesthimani Misirli. The syndrome of rhabdomyolysis: Pathophysiology and diagnosis. European Journal of Internal Medicine. 2007;18:90-100.

[5] Delrio FG, Park Y, Herzlich B, Grob D. Case report: diclofenac-induced rhabdomyolysis. Am J Med Sci 1996; 312:95-7.

[6] Knobloch K, Rossner D, Gössling T, Lichtenberg A, Richter M, Krettek C. Rhabdomyolysis after administration of diclofenac. Unfallchirurg 2005; 108:415-7.

[7] Ahmet Güzel, Betül Orhaner Biner, Serap Karasalihoğlu,
Hakan Aylanç. Fatal Acute Diclofenac-Induced Rhabdomyolysis in A Pediatric Patient. Balkan Med J 2011; 28: 102-103.

[8] Mahmood Al kindy, Vidia Limaye, Pravin Hissaria. Meloxicam induced rhabdomyolisys in the context of acute cross river infection. Allergy Asthma Immunol Res.2012 January 4(1); 52-54.

[9] Teresa J. Coco and Ann E. Klasner. Drug-induced rhabdomyolysis. Current Opinion in Pediatrics.2004; 16: 206-210.

[10] Kallel H, Chelly H, Dammak H, Bahloul M, Ksibi H, Hamida $\mathrm{CB}$, et al. Clinical Manifestations of systemic paraphenylene diamine intoxication. J Nephrol 2005;18:308-11.

[11] Krishnaswamy Sampathkumar, Yesudas Santhakumari Sooraj, Rajappannair Prabha Ajeshkumar, Amol Ramesh Mahaldar, Ramakrishnan Muthiah. Rhabdomyolysis due to hair dye poisoning: An emerging threat. Indian Journal of critical care medicine. 2007; 11(4):212-214.

[12] Cervellin G, Comelli I, Lippi G. Rhabdomyolysis: historicalbackground, clinical, diagnostic and therapeutic features. Clin Chem Lab Med 2010;48(6):749-56. 